\title{
Use of Plasmid pVMG to Make Transcriptional ß-Glucuronidase Reporter Gene Fusions in the Rhizobium Genome for Monitoring the Expression of Rhizobial Genes In Vivo
}

\author{
Mengsheng Gao*, Anne Benge, Tai-Jung Wu and Regina Javier
}

\begin{abstract}
Background: The soil bacterium Sinorhizobium meliloti and its allies are important nitrogen-fixing bacterial symbionts that cause $\mathrm{N}_{2}$-fixing nodules on the roots of legumes. Chromosomal B-glucuronidase gene (uidA) transcriptional fusions are frequently used to monitor the expression of bacterial genes during the symbiosis. However, the construction of the fusions is laborious.

Results: The narrow-host-range, fusion selective plasmid pVMG was constructed and used as a vector for the construction of chromosomal uidA transcriptional fusions in the S. meliloti genome. Translation termination codons were added in all three reading frames upstream of the promoterless uidA in this vector to ensure transcriptional fusions. pVMG replicated to high copy number in Escherichia coli, offering advantages for the isolation of fusion-containing plasmids and the restriction analysis. Genomic locations of uidA fusions were verified in a simple PCR experiment. All these helps reduce the sample processing time and efforts. As a demonstration of its usefulness, the $\mathrm{N}$-acyl homoserine lactone (AHL) signal synthase gene promoter was fused to uidA and shown to be expressed by $S$. meliloti in the senescence zone of the nodule on the host plant, $M$. truncatula. This indicates the presence of AHL signals at the late stages of symbiosis.
\end{abstract}

Conclusions: A simple, pVMG-based method for construction of chromosomal uidA transcriptional fusions has been successfully used in the model rhizobium S. meliloti. It is also applicable for other rhizobial strains.

Keywords: Rhizobia symbiosis, B-glucuronidase gene (uidA), Transcriptional fusions, Chromosomal uidA transcriptional fusions

\section{Background}

The chromosomal uidA transcriptional fusions are frequently used for monitoring in vivo expression of bacterial genes for at least three reasons: First, the fusions allow the transcriptional activities of bacterial genes to be monitored at their nature levels [1-4]. Second, the fusions do not need antibiotics for maintaining their stability in the genome. Third, they avoid

\footnotetext{
* Correspondence: msgao@ufl.edu

Soil and Water Sciences Department, Cancer and Genetics Research

Complex, University of Florida-Institute of Food and Agricultural Sciences,

Room 330E, Gainesville 32610, USA
}

problems that associate with replicating plasmid systems which can disrupt regulation of expression due to copy number effects $[1,3-5]$. As higher plants lack ß-glucuronidase activity [6], the uidA gene provides a sensitive enzyme assay for which a broad range of substances are available.

Methods for making chromosomal uidA transcriptional fusions in rhizobia involve a bacterial narrow-host-range, plasmid vector with a promoterless $\operatorname{uidA}$ gene $[1,2,4]$. Segments of DNA containing gene promoter from rhizobia can be cloned into the multiple cloning sites (MCS) located upstream of the uidA in the vector. The 
fusion-containing plasmids are maintained in a suitable E. coli strain, isolated and restriction analyzed, and then can be transferred to a rhizobial strain from $E$. coli in bi- and tri-parental mattings. As the vector uses an origin of replication (e. g., pUC) that is inactive in rhizobial strains [7], each fusion-containing plasmid co-integrates into the rhizobial host with the rhizobial host DNA. This can create a single copy uidA transcriptional fusion in the rhizobial host genome. The genomic locations of the fusions are typically verified by Southern blotting. The integration of fusion-containing plasmid does not disrupt the targeted locus if the cloned DNA fragment in the plasmid will not be internal to the transcription unit $[1,8]$.

A few plasmids have been used as narrow-host-range uidA transcriptional vectors in pioneering studies of rhizobial gene expression: pMH11 [9], pVO155 [1, 2], and pTH1522 [4]. While very fruitful to make uidA transcriptional fusions for in vivo studies, some of those vectors lack translational termination codons between their MCS and promoterless uidA reporter gene, therefore require additional experiments to ensure transcriptional fusions. Others are low copy number plasmids which can make restriction analysis and isolation ineffective. In some of those methods, Southern blotting was used to verify genomic locations of uidA fusions. While specific and sensitive, Southern blotting is timeconsuming. Therefore, we have constructed a transcriptional uidA fusion selective plasmid pVMG on the basis of pVO155 [1] and developed a pVMG-based method for the construction of chromosomal uidA transcriptional fusions in the rhizobial genome. We showed that PCR has the necessary combination of simple procedure, sensitivity and consistent results thus can be used in the place for verifying uidA fusions. We demonstrated the utility of the pVMG and the pVMG-based method by constructing and testing a transcriptional fusion between the sinI gene of $S$. meliloti and uidA.

\section{Methods}

\section{Bacterial Strains and Media}

All strains and plasmids used are listed in Table 1. The following media were previously described: LB, a complex medium [13]; TY, a complex medium for S. meliloti [14], containing, Per liter, tryptone $6 \mathrm{~g}$; yeast extract $3 \mathrm{~g}$, and $\mathrm{CaCl}_{2} \cdot 2 \mathrm{H}_{2} \mathrm{O} \quad 0.5 \mathrm{~g}$. Final concentrations of antibiotics: $100-200 \mathrm{\mu gml}^{-1}$ of neomycin (Nm) and 250-500 $\mu_{\mathrm{gml}}{ }^{-1}$ of spectinomycin (Sm) (for the S. meliloti strains).

\section{Biochemistry \\ DNA Biochemistry}

Restriction endonucleases were purchased from New England Biolabs (New England Biolabs, Inc. Beverly, MA, USA) and used according to the manufacturer's instructions. A 25-bp linker was created by annealing two complementary oligonucleotides (5' - GATCCCTCGAG CTGACTAACTAGCT-3'; 5'CTAGAGCTAGTTAGTCA GCTCGAGG-3'). The linker contained a Xho I site and translation termination codons in three different ORFs with termini cohesive to either BamH I or Xba I. DNA ligations were performed with T4 DNA ligase (Biolabs, \# M0202 T). Colony PCR was performed in a sterile 0.5-ml amplification tube containing 1 x Standard Taq Reaction Buffer (Biolabs \#B9014S), $0.5 \mu \mathrm{M}$ of four dNTPs, $0.2 \mu \mathrm{M}$ of two forward and revers primers, 1 unit of Taq DNA polymerase (Biolabs \#M0320 L) and an individual $S$.

Table 1 Strains and plasmids

\begin{tabular}{|c|c|c|}
\hline Strain or plasmid & Relevant features & Source or Reference \\
\hline \multicolumn{3}{|l|}{ Strains } \\
\hline $\mathrm{DH} 5 \mathrm{a}$ & a-complementation & Invitrogen \\
\hline 8530 & S. meliloti, $\exp R+, \sin 1+, \mathrm{Sm}^{r}$ & [10] \\
\hline 1021 & S. meliloti, $\exp R-, \sin l+, \mathrm{Sm}^{\mathrm{r}}$ & [11] \\
\hline MG32 & 8530 with $\sin /$ deletion $\exp R+, \sin \Gamma, \mathrm{Sm}^{r}$ & [12] \\
\hline 8530 sinl::VMG495 & $\operatorname{sinl}$-uidA transcriptional fusion, $\exp R+, \sin /+, \mathrm{Sm}^{r}, \mathrm{Nm}^{r}$ & This work \\
\hline 1021 sinl::VMG495 & $\operatorname{sinl-uidA}$ transcriptional fusion, $\exp R+, \sin /+, \mathrm{Sm}, \mathrm{Nm}$ & This work \\
\hline MG32 sinl::VMG495 & $\operatorname{sinl}$-uidA transcriptional fusion, $\exp R+, \sin l-, \mathrm{Sm}^{r}, \mathrm{Nm}^{r}$ & This work \\
\hline 8530 nop::VMG209 & Non promoter (nop) DNA-uidA, expR+, $\sin /+, \mathrm{Sm}^{\mathrm{r}}, \mathrm{Nm}^{\mathrm{r}}$ & This work \\
\hline \multicolumn{3}{|l|}{ Plasmids } \\
\hline pV0155 & pUC19-derived integrational uidA vector & [1] \\
\hline pVMG & pVO155 with stop codons upstream of promoterless uidA in all ORFs & This work, [12] \\
\hline pRK600 & pRK2013 Nm::Tn9, $\mathrm{Cm}^{r}$ & [1] \\
\hline pVMG495 & pVMG, sin/ 5'-end, transcriptional fusion & This work \\
\hline pVMG209 & pVMG, nop DNA of S. meliloti. & This work \\
\hline
\end{tabular}


meliloti colony. PCR primers are shown in Table 3. The nucleic acids were amplified for 35 cycles. The denaturation, annealing, and polymerization times and temperatures were $1 \mathrm{~min}$ at $94^{\circ} \mathrm{C}, 1 \mathrm{~min}$ at $50^{\circ} \mathrm{C}$, and $30 \mathrm{~s}$. at $72^{\circ} \mathrm{C}$. DNA sequencing was performed by Rightmire DNA Sequencing Facility at The Ohio State University, Columbus, Ohio, and by the DNA Sequencing Laboratory at the Interdisciplinary Center for Biotechnology Research at University of Florida, Gainesville, Florida.

Genomic DNA extraction was described previously [4] with minor modifications. DNA was prepared from $5 \mathrm{ml}$ of culture grown in TY broth with appropriate antibiotics to saturation with shaking. Bacteria were collected in 2-ml microcentrifuge tubes by centrifugation, washed once with $0.85 \% \mathrm{NaCl}$, and then resuspended in $750 \mu \mathrm{l}$ of TE buffer (10 mM Tris- $\mathrm{HCl}, \mathrm{pH} 8.0, \mathrm{mM}$ EDTA). Sodium dodecyl sulfate (SDS) was added to $1 \%, \mathrm{NaCl}$ to $1 \mathrm{M}$, and proteinase K (Sigma) to $0.5 \mathrm{mg} / \mathrm{ml}$. Samples were mixed gently and incubated at $65{ }^{\circ} \mathrm{C}$ for $2 \mathrm{~h}$ for complete lysis. DNA was extracted twice with equal volume of buffer-saturated phenol, twice with 1:1 phenolchloroform, and once with chloroform, and then $\mathrm{NaCl}$ was added to $0.2 \mathrm{M}$ and nucleic acids precipitated with a 2.5 volumes of ethanol. The pellet was dissolved in $400 \mu \mathrm{l}$ of TE with $20 \mathrm{~g} / \mathrm{ml}$ RNase A and incubated for $30 \mathrm{~min}$ at $37^{\circ} \mathrm{C}$. DNA was extracted once with 1:1 phenol-chloroform and once with chloroform, ammonium acetate was added to $0.5 \mathrm{M}$, and DNA was precipitated with an equal volume of isopropanol. The optical density at $260 \mathrm{~nm}\left(\mathrm{OD}_{260}\right)$ of the sample was measured to determine the DNA concentration. The samples were then lyophilized and dissolved to proper concentrations for restriction enzyme digestions.

\section{Acyl Homoserine Lactones (AHL) Biochemistry}

$\mathrm{C}_{16: 1}-\Delta^{9}$ cis-(L)-homoserine lactone (referred to as " $\mathrm{C}_{16: 1}-\mathrm{HSL}$ " in text) was from Cayman Chemical (Ellsworth Road, Ann Arbor, MI U.S.A.). The molecule was dissolved in ethyl acetate at stock and diluted in methanol. AHL was added in a liquid medium prior to inoculation of bacteria.

\section{B-Glucuronidase (GUS) Biochemistry}

Quantitative assay for GUS activity was described previously [6] with modification [15]. Cells were permeabilized with lysozyme ( $200 \mathrm{\mu g} \mathrm{ml}^{-1}, 37^{\circ} \mathrm{C}$ for $\left.10 \mathrm{~min}\right)$, and the GUS activity was measured with PNPG (p-nitrophenyl- $\beta$-o-glucuronide). GUS activity was calculated in nanomoles per minute per $\mathrm{OD}_{595}$ unit $\times 1000$ as in [15]). Color producing substrate X-gluc (5-brome-4-chloro-3-indolyl- $\beta$-D-glucuronide) for GUS enzyme to act was used for visualizing activity of GUS in histochemical assay at working concentration of $40 \mu \mathrm{g} / \mathrm{ml}$ as described before [16].

\section{Conjugations}

Recombinant plasmids were maintained in E. coli DH5 $\alpha$ and were conjugated into $S$. meliloti recipient strains with help plasmid pRK 600 by bacterial conjugation method [1] with modifications. Log-phase recipient cells $\left(10^{+8} / \mathrm{ml}\right)$ were used in the conjugation. The donor and recipient ratio was approx. 8:1. S. meliloti transconjugants were selected on TY agar medium at the present of neomycin.

\section{Sequence Analysis}

Sequence assembly was performed with MacVector with Assembler 12.01. Database searches were conducted through the $S$. meliloti genome web page using blastn.

\section{Plant Growth and Nodulation}

Cultivation of $M$. truncatula A17, root nodulation, and nodule harvesting were described previously [17].

\section{Results \\ Construction of pVMG}

The narrow-host-range plasmid pVO155, with a MCS, a promoterless uidA (gus) reporter gene encoding ß-glucuronidase (GUS), and a pUC origin of replication, was used as a base for the construction of pVMG.

pVMG was constructed by replacing a BamH I-Xba I fragment at the end of MCS in pVO155 with the 25-bp $B a m \mathrm{H}$ I-Xba I synthetic DNA linker containing three translation terminations and a Xho I site (see Methods). The structure of pVMG is shown in Fig. 1a. The translation termination codons (referred to as to "stop codons" in Figure) upstream of the uidA in all reading frames ensure transcriptional fusions (Fig. 1b). DNA sequencing and double/triple endonuclease digests of the plasmid confirmed that $\mathrm{pVMG}$ retains the original promoterless uidA gene, seven of the 8 restriction enzymes in multiple cloning sites (MCS), a E. coli $\operatorname{trp} A$ terminator upstream of the MCS to prevent read-through from the vector, a unique Hind III site adjacent to the $\operatorname{trp} A$, a unique $B g l$ II and the pUC origin of replication. DNA sequencing data also confirmed that pVMG retains the original oriT origin of transfer, the neomycin $(\mathrm{Nm})$ resistance ORF and the ampicillin (Amp) resistance ORF.

pVMG replicates to high copy number in E. coli DH5a. This provides cloning advantages. The average DNA yield of pVMG in Miniprep experiments was similar to the yield of pUC19 itself (Table 2). Various 200-800 bp DNA fragments from S. meliloti were cloned into pVMG $[12,18,19]$. For an insert size of $200-800 \mathrm{bp}$, we typically obtained $80-190 \mathrm{Nm}^{\mathrm{R}}$ colonies by using the $1 / 5$ volume of each ligation reaction and of these, approximately $85 \%$ contained the expected inserts. 


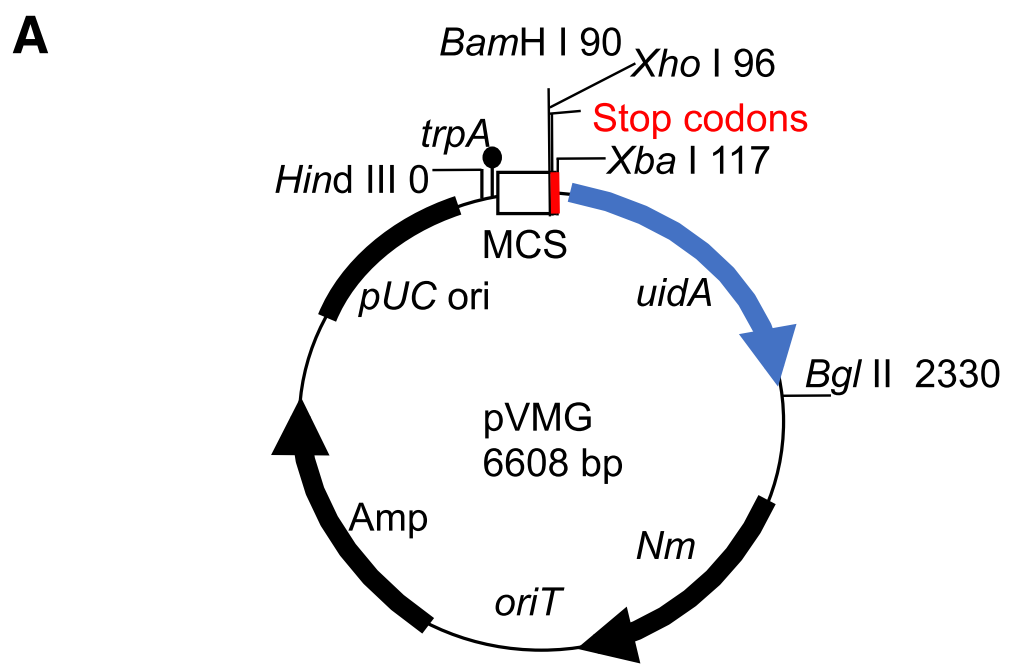

B

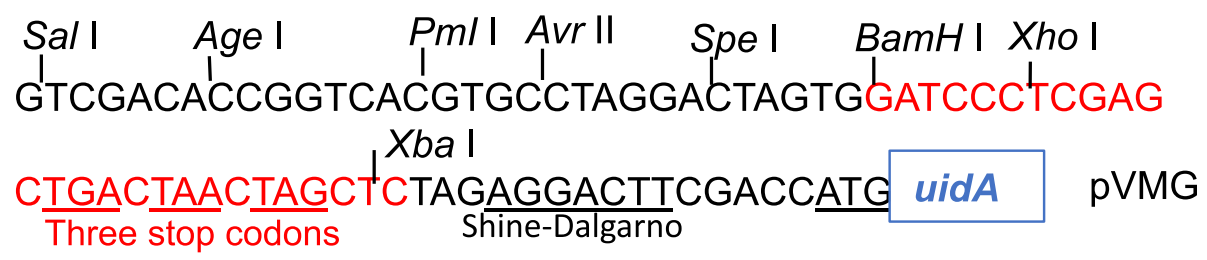

Fig. 1 Structure and multiple cloning sites (MCS) of the plasmid pVMG. a. pVMG, a derivative of pVO155, contains a short DNA linker (red) for translation termination codons to ensure transcriptional fusions. The size of pVMG was deduced from the known sequence of the analogous plasmid pVMGTnpR (GenBank accession number EU232661). b. The MCS of pVMG. All the restriction sites are usable except the Xba I site. The $\mathrm{Xba} \mathrm{I}$ is located after translation termination codons, therefore not readily usable for making transcriptional fusions

\section{Construction of Transcriptional sinl-uidA Fusions}

In order to demonstrate the usefulness of pVMG, we made a transcriptional sinI gene reporter fusion to uidA by using a pVMG-based method (Fig. 2a). The sinI gene of $S$. meliloti encodes the synthase of the bacterial AHL signaling molecules [20]. The sinI gene mutations abolish the transcription of sinI-dependent genes and delay initiation of nodulation on the roots of the host plant, $M$. truncatula [12]. The sinI promoter is inducible by $\mathrm{C}_{16: 1}$-HSL and the ExpR protein of S. meliloti enhances

Table 2 DNA yields of pVMG, pVO155 and pUC19 grown in medium LB

\begin{tabular}{ll}
\hline Plasmid & Average yield $n=$ exp. (Mg) \\
\hline pUC19 & $18 \pm 3(n=5)$ \\
pVMG & $16 \pm 2(n=75)$ \\
pVO155 & $16 \pm 2(n=17)$ \\
\hline
\end{tabular}

QIAprep Spin Miniprep Kit was used to purify DNA from $1.5 \mathrm{ml} \mathrm{LB}$ overnight cultures of DH5a containing pUC19, or pVMG or pVO155. Elution was performed according to the standard protocol (50 $\mu \mathrm{l} \mathrm{Buffer} \mathrm{EB}$ and $1 \mathrm{~min}$ incubation). Use of the recommended LB composition (with $10 \mathrm{~g} /$ liter $\mathrm{NaCl}$ ) and $\mathrm{Nm}$ resistance provides optimal plasmid yield. Time spent was $<15 \mathrm{~min}$. Costs per $\mu \mathrm{g}$ DNA was 7 cents. this induction [10]. The $\operatorname{sinI}$ gene is expressed in free-living bacteria and at the time when cells invade the nodule of $M$. truncatula [12]. In our study of the Rhizobium sinI gene function, we wished to examine the expression of $\sin I$ gene in late stages of the symbiosis.

The intact 5'-end of $\sin I$ gene fragment was cloned into pVMG. This 438-bp fragment contains the sinI promoter and ExpR binding site (nucleotides -416 to +22 with respect to the $\sin I$ translation start site) [21]. The fragment was amplified from chromosome of S. meliloti. The oligonucleotide primers (Primer 1-sinI and Primer 2 -sinI) used for the amplification are shown in Table 3. The Primer 1 contains a Sal I site and the primer 2 contains a $B a m \mathrm{H}$ I site. The PCR-amplified fragment was treated with $\mathrm{Sal}$ I and $B a m \mathrm{H}$ I, then ligated to the Sal I-BamH I digested pVMG. This ligation created a transcriptional sinI-uidA fusion-containing plasmid pVMG495. pVMG495 was transferred into Invitrogen DH5a competent cells which are neomycin sensitive $\left(\mathrm{Nm}^{\mathrm{S}}\right)$.

pVMG495 was conjugated into the $S$. meliloti expR mutant strain 1021 [11] by a tri-parental matting with pRK600 as a helper. S. meliloti conjugants were selected 


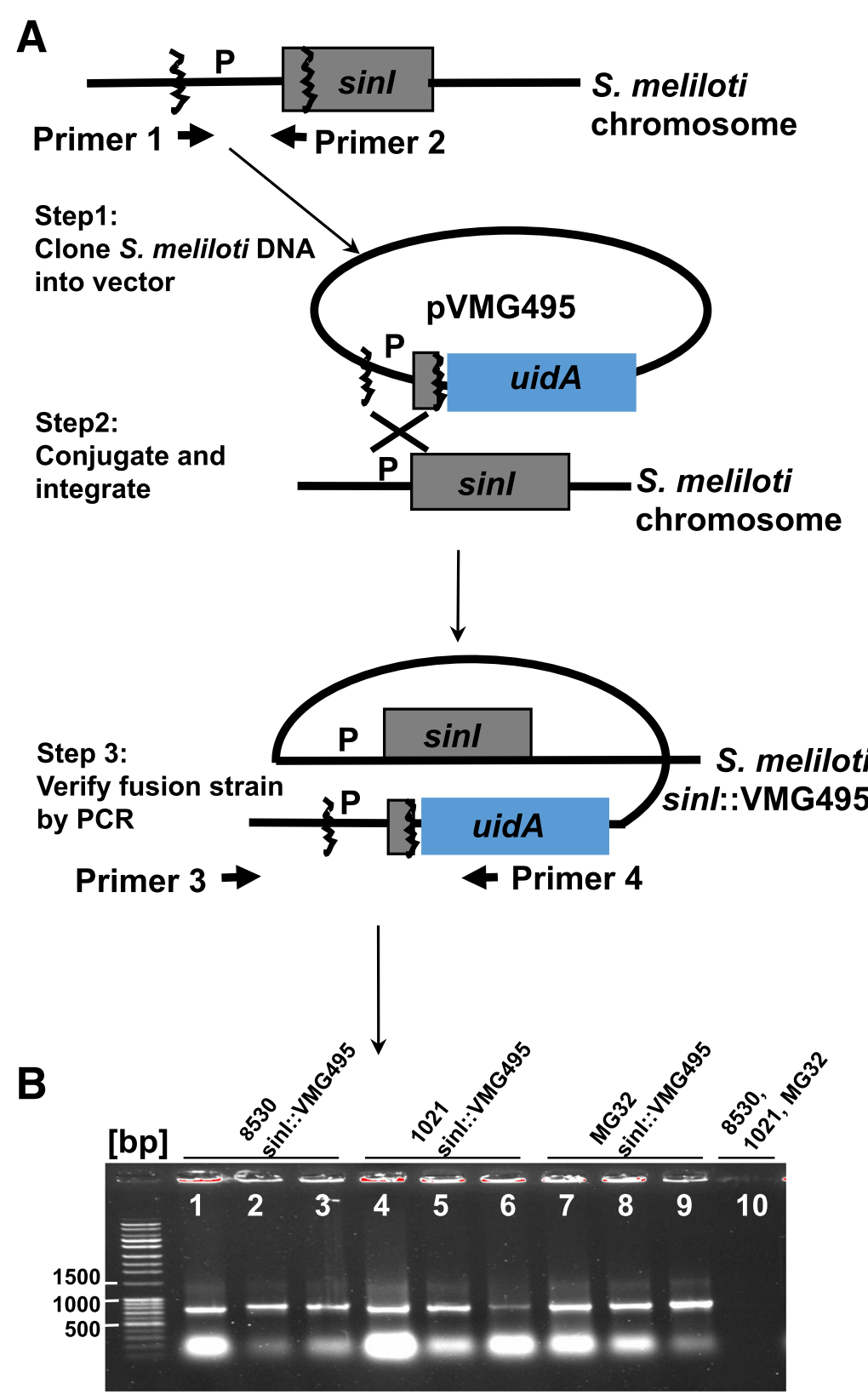

Fig. 2 Testing the pVMG method. a. pVMG method overview. b. Gel electrophoresis of PCR-amplified 720-bp DNA. 720-bp PCR-bands were specific for S. meliloti reporter fusion strains 8530 sinl::VMG495, MG32 sinl::VMG495, and 1021 sinl::VMG495. DNA marker: The Invitrogen Tracklt 1 Kb Pluse DNA Ladder

for neomycin resistance $\left(\mathrm{Nm}^{\mathrm{R}}\right)$, yielding a transcriptional sinI-uidA fusion strain called S. meliloti 1021 sinI::VMG495 (Table 1).

In the experiments reported above, the co-integration frequency of the plasmid pVMG with the 438-bp chromosomal insert in the S. mlilloti 1021 was about $4.3 \times 10^{-5}$ (number of $\mathrm{Nm}^{\mathrm{r}}$ recombinants per total number of receipted cell present). This represents about a 8.6-fold increase over the co-integration frequency obtained in Azorhizobium caulinodans ORS571 [22] for a pBBR-based replicon with a larger insert introduced by a similar tri-parental matting method. In a control experiment, the vector pVMG was conjugated into the strain 1021, no $\mathrm{Nm}^{\mathrm{r}}$ - transformants were obtained.

Two additional transcriptional $\operatorname{sinI}$-uidA fusion strains were constructed by conjugating pVMG495 into S. meliloti sinI mutant strain MG32 [12] and S. meliloti wild-type strain 8530 [10], respectively. They yielded S. meliloti MG32 sinI::VMG495 and S. meliloti 8530 sinI::VMG495 (Table 1). 
Table 3 Primers used in the study. Sal I and BamH I restriction sites are underlined.

\begin{tabular}{lll}
\hline Name & Sequence (5'-3') & usage \\
\hline Primer 1-sinl & ACGCGTCGACGTTGAGTGGTCCGCCTACCG & sinl forward \\
Primer 2-sinl & GCTGCGACCGGATCCGTTCACTAT & sinl reverse \\
Primer 3-sinl & GGTGGAATGGGCGACAGCGCG & $\sin /$ forward \\
Primer 4 & GGGTTGGGTTTCTACAGGA & uidA reverse \\
Primer 1-nop & AGCCTTGAACGTCGACTG & nop forward \\
Primer 2-nop & ATGGAGGATCCAGCGAG & nop reverse \\
Primer 3-nop & AAGTTGGGGTATCGCCCTAAA & nop forward \\
\hline
\end{tabular}

All S. meliloti sinI-uidA fusion strains (1021 sinI::VMG495, MG32 sinI::VMG495, 8530 sinI::VMG495) were verified for the site-specific integration of pVMG495 by PCR. Three colonies of each fusion strain candidates were analyzed. The oligonucleotide primers (Primer 3-sinI and Primer 4) used for the amplification are shown in Table 3. The integration specific Primer 3-sinI attaches the genomic DNA region outside and upstream of the cloned 438-bp fragment. The uidA specific primer 4 attaches DNA region inside of the uidA from pVMG and it faces toward the cloned $\sin I$ fragment (Fig. 2a). Specific primers amplified the $\sim 720$-bp DNA region of integration (Fig. 2b, lanes 1-9) from all tested fusion strains, but not from their parental strains (Fig. 2b, lane 10). The fusion-strain-specific amplification strongly suggests the site-specific integration of pVMG495. The 720-bp product was presumably constituted of the cloned $\sin I$ fragment flanked by the upstream $S$. meliloti chromosome and the 5 -end of uidA gene. The identity of the 720-bp product was determined by DNA sequencing. This confirmed the presence of all expected DNA segments as well as a termination codon in-frame with the $\sin I$ gene (see Additional file 1).

Next, we checked the identity of S. meliloti reporter strains (1021 sinI::VMG495, MG32 sinI::VMG495 and

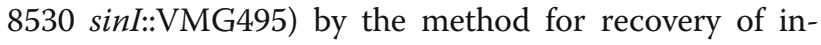
tegrated plasmid for subsequent DNA sequencing $[1,8]$. We cut the entire genome with a restriction enzyme that does not cut within the pVMG495 plasmid. We then circularized the fragments with $\mathrm{T}_{4}$ DNA ligase and transformed them into E. coli strain DH5a. We recovered a plasmid that contains the expected structure in every strain.

Subsequently, we checked the identity of $S$. meliloti reporter strains (1021 sinI::VMG495, MG32 sinI::VMG495 and 8530 sinI::VMG495) by Southern blot [23]. As shown in Additional file 2 Figure S2, Southern blot test detected one fragment of Hind III digestion in the DNA from each sample of the fusion strains of sinI::VMG495. The identical band was visible at approx. 7.7-kb from each fusion sample since the $\sin I$ gene integration regions are identical. This band was caused by the chimeric DNA fragment of genome-pVMG495. The Southern blotting test detected no additional second or third copies of the vector in the genomic DNA samples, suggesting no random integrated vector DNA in the genomes of $1021 \sin I:$ VMG495, MG32 sinI::VMG495 and 8530 sinI::VMG495.

By using the pVMG method shown in Fig. 2a, we constructed a control strain called S. meliloti 8530 nop::VMG209 (Table 1) for measuring background activity of the GUS. In this experiment, a plasmid called pVMG209 was created by cloning a 209-bp non-promoter (nop) DNA segment of S. meliloti to the SalI-BamHI site of pVMG. pVMG209 was integrated into the chromosome of the S. meliloti 8530 strain. The primers we used for the construction of 8530 nop::VMG209 strain are shown in Table 3.

\section{Testing Transcriptional sinl-uidA Fusions}

The fusion strains and the control strain were tested for responsive changes in GUS activity in free-living bacteria. The rhizobium strains were cultured in (a) TY broth, (b) TY broth containing $7.5 \mathrm{nM}$ of $\mathrm{C}_{16: 1}$-HSL as we did before [24]. The fusion in $\sin I$ promoter was found to have significant changes in GUS activity in response to $\exp R \sin I$, $\sin I$, $\exp R$ backgrounds or to added AHL when tested at late log phase (optical density at $600 \mathrm{~nm}\left[\mathrm{OD}_{600}\right]=0.98$ to 1.08 .) (Table 4). Quantitative GUS assay demonstrated that the $\operatorname{sinI}$ promoter activity in the presence of $C_{16: 1}$ was 5.8-fold higher than in the absence of $\mathrm{C}_{16: 1}$. This induction factor is consistent with the one measured in a $S$. meliloti reporter strain carrying a chromosomal single copy of $\sin I-l a c Z$ transcriptional fusion [25]. The uidA fusion in non-promoter (nop) DNA had no activity in WT background (Table 4) and was not responsive to the AHL (data not shown).

The wild-type strain that expresses $\beta$-glucuronidase (GUS) was also tested for its ability to initiate nodulation on roots of $M$. truncatula plants, using a previously described protocol [17]. The rate and the efficiency of

Table 4 Responses of single copy sinl-uidA transcriptional fusion to $\exp R \sin 1, \sin l, \exp R$, and $A H L$ in medium $T Y$

\begin{tabular}{lll}
\hline Strain & Genotype & $\begin{array}{l}\text { B-glucuronidase } \\
\text { activity }\end{array}$ \\
\hline 8530 sinl::VMG495 & expR+, sinl+ & $194 \pm 4$ \\
MG32 sinl::VMG495 & expR+, sinl- & $33 \pm 3$ \\
MG32 sinl::VMG495 $\left(C_{16: 1}\right)$ & expR+, sinl- & $192 \pm 2$ \\
1021 sinl::VMG495 & expR-, sinl+ & $95 \pm 3$ \\
8530 nop::VMG209 & expR+, sinl+ & $<0.1$ \\
\hline
\end{tabular}

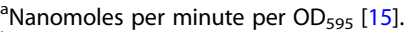

${ }^{\mathrm{b}}$ Shown are averages and standard deviations $(n=3)$. 
nodule initiation by the GUS expressing strain 8530 sinI::VMG495 and the 8530 parent were found similar. This observation indicated that 8530 sinI::VMG495 was normal in the ability to initiate nodulation on the host plant. The normal initiation of nodulation suggested that $\sin I$ activity was not inhibited by the GUS or by the $\sin I$ fragment of 8530 sinI::VMG495 strain.

In order to examine the expression of $\sin I$ gene in the late stages of the symbiosis, roots of $M$. truncatula were inoculated with 8530 sinI::VMG495 and 8530 nop::VMG209. Nodules were harvested and stained with X-Gluc [6]. Stained nodules were examined under a dissecting scope. The 8530 sinI::VMG495 infected nodules showed a blue invasion zone and a blue senescence zone (Fig. 3a). The 8530 nop::VMG209 infected nodules did not (Fig. 3b). While confirming the expression of the $\sin I$ by $S$. meliloti bacteria at the time of nodule invasion [27], the results demonstrated the expression of the $\sin I$ in the senescence zone of the $M$. truncatula root nodule. This indicates the presence of AHL signals at the late stages of symbiosis.

\section{Discussion}

Several reporter gene systems are available to monitor bacterial gene activity within plant cells. These include $\beta$-galactosidase (lacZ) [28], green fluorescent protein (gfp) [29] and $\beta$-glucuronidase (uidA) [6]. uidA reporter gene system has increased sensitivity relative to lacZ because most higher plants show large amounts of galactosidase activity but they lack $ß$-glucuronidase (GUS) activity [6]. uidA reporter system has increased sensitivity relative to GFP when used within root nodule cells because molecular oxygen $\left(\mathrm{O}_{2}\right)$ is low in those cells but GFP strictly requires $\mathrm{O}_{2}$ for maturation of fluorescence [30]. Because it has increased sensitivity, uidA has been extensively used to monitor bacterial gene activity during the Rhizobium-legume symbiosis [1, 2, 4, 19, 31, 32].

In the course of research in S. meliloti, we have developed a new transcriptional uidA fusion selective vector that preserves properties of its parent plasmid pVO155, i.e., to be a mobilizable narrow-host plasmid and present at a high copy number in E. coil. The characteristic presence of translation terminations between the MCS and the promoterless uidA gene in a pUC-based replicon, distinguishes pVMG as an effective vector to make chromosomal uidA transcriptional fusions in Rhizobia. This effectiveness of making transcriptional fusion, together with the simple procedure of verifying fusion by PCR, distinguish pVMG method as an effective method for various applications in construction gene fusions of Rhizobium. We have found that Rhizobium DNA fragments with size up to $3.5-\mathrm{kb}$ can be easily cloned in pVMG [12, 18, 19] and yields of those Rhizobium
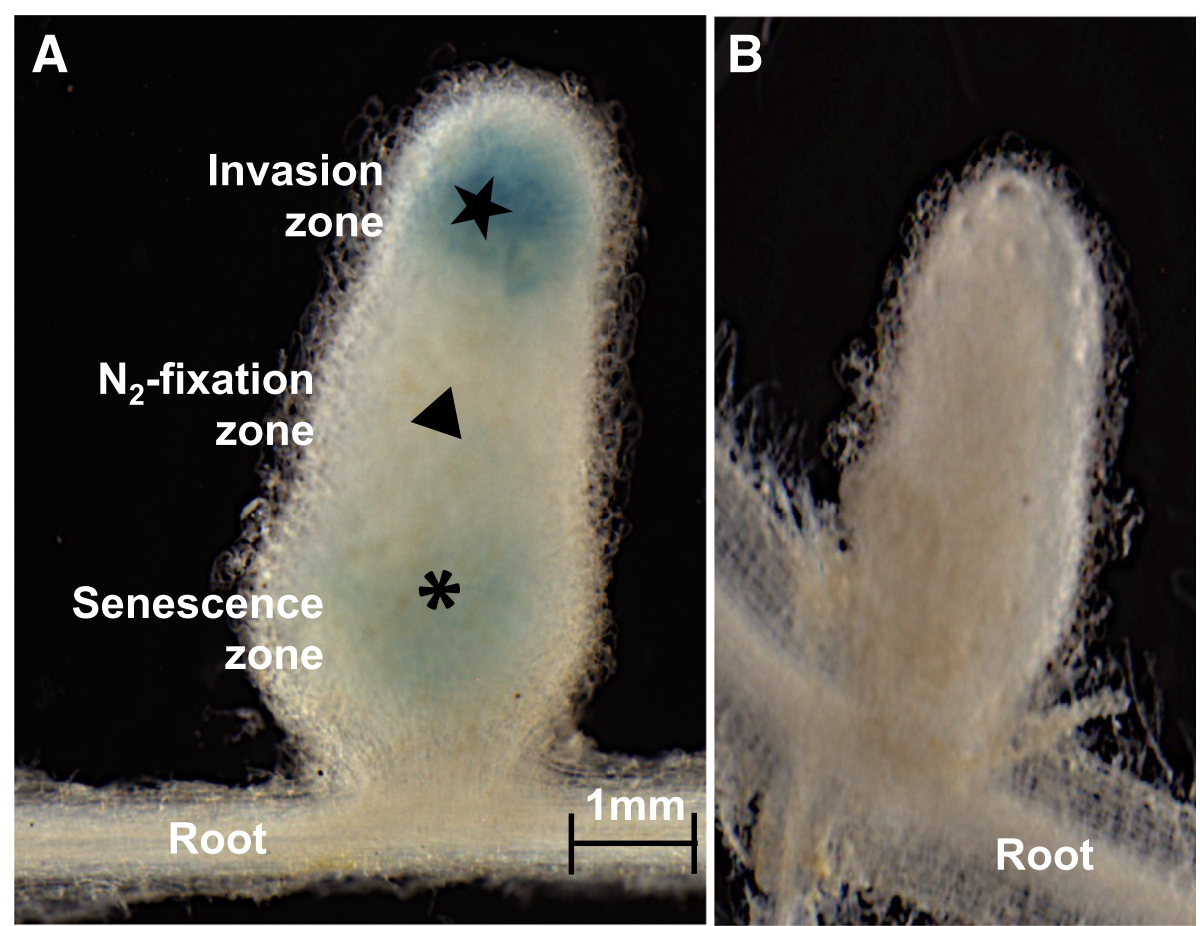

Fig. 3 Histochemical assay of M. truncatula nodules. a 8530 sinl::VMG495 $(n=13)$ b 8530 nop::VMG209 $(n=5)$. Nodules were stained with 4 mg $/ \mathrm{ml} \mathrm{X}$-Glue in $\mathrm{NaH}_{2} \mathrm{PO} 4 . \mathrm{pH} 7.2,37^{\circ} \mathrm{C}$ as described [26] for $3 \mathrm{~h}$ (a) or for $48 \mathrm{~h}$ (b). Photographs were taken 7 weeks after inoculation with Olympus MVX10 dissecting scope equipped with a MicroFire camera (Optitronics, Goleta, CA, USA) 
fusion-containing plasmids from minipreps were similar to the yield of pUC19 which is much higher than those of pBBR-based narrow-host plasmids. Large amount of candidate rhizobial uidA fusion strains can be verified easily by a simple one-step colony PCR which is much easier than Southern blotting or recovery of integrated plasmid for verification of fusion. Additionally, a PCR product could be used to identify the genomic site of integrated plasmid by direct DNA sequencing.

Genomic sites of integrated plasmid have been typically verified with the Southern blotting procedure [23]. Southern blotting is extremely sensitive and specific for detecting DNA in a particular sample and has been used in diagnostic studies to detect genomic sites of integrated plasmid in bacteria [2, 12, 22, 33, 34]. Although sensitive and specific, Southern blotting requires isolation of DNA, digestion of DNA with restriction enzyme (s), separation of DNA by agarose gel electrophoresis, blotting and hybridization with a probe [16]. Making a probe for hybridization involves generating, purifying and labeling DNA. In most cases, radioactive probes are used [16]. While generally effective, these methods are time consuming and create the potential for radiation exposure.

In an effort to reduce sample processing time and efforts, we have verified the genomic site of the integrated plasmid by the simple PCR experiment for subsequent DNA sequencing. Results from this verification method were checked with other two conventional verification methods: 1) the method for recovery of integrated plasmid for genome-vector site identification $[8,35]$ and 2) Southern blotting [23]. Results from both conventional verification methods supported the conclusion that the PCR verification method has the necessary combination of simple procedure, sensitivity and specificity. Thus, the introduction of the PCR verification method can reduce the use of Southern blotting in diagnostic studies for verification of genomic sites of integrated plasmid.

As shown in Fig. 2a, the genome of S. meliloti uidA reporter strain contains the vector DNA. Because the vector DNA has not affected any known genes for the symbiosis and does not appear to affect the growth of the bacteria, the symbiotic behaviors of the reporter strain are as normal as its parental strain. When one wishes to use pVMG to make a chromosomal uidA fusion while avoiding the vector DNA, one possibility becomes apparent. It is possible to use tnpR encoded resolvase [36] to excise the vector DNA from the genome. The resolvase catalyzes excision of the DNA flanked by short DNAs called res sequences. pVMG (Fig. 1a) has a unique HindH III site upstream of the MCS and a unique $B g l$ II site downstream of uidA, allowing insertion of the res sequences for the resolvase to function. Similar types of work have been successfully carried out in S. meliloti for other target region excision purposes and a version of pVMG carrying a inducible tnpR exists [3]. Other techniques might be of use. In CRISPR-Cas9, for example, the class of RNA-guided endonucleases known as Cas9 from the microbial adaptive immune system CRISPR (clustered regularly interspaced short palindromic repeats) can be targeted to virtually any genomic location of choice by a short RNA guide [37]. With two such guides, a CRISPR-Cas9 system has generated target excisions in the genomes of bacteria Streptococcus pneumoniae and E. coli [38]. Given the results of work with those bacteria, CRISPR-Cas9 might be of use to excise the vector DNA from the genome of S. meliloti.

In addition to the generation of transcriptional uidA fusions in a DNA region from $S$. meliloti, we have been using pVMG for generating genome-wide transcriptional uidA fusions in the bacterium. We have cloned a library of the S. meliloti DNA fragments to the BamHI site of pVMG and screened the resulting strains for the sinI-regulated activity of transcription in the late stages of symbiosis. Preliminary studies of transcription of S. meliloti genes linked expression of some of these genes to the activity of the $\sin I$ gene (unpublished results). In fact, the analogous plasmid pVO196 [1] has already been successfully used for generating a library of S. meliloti transcriptional fusions to a promoterless copy of $b a c A$ gene for discovering activity of the rhizobial genes in the intermediate stages of the symbiosis. According to our preliminary studies and the published study, it is likely that pVMG will be useful for the in planta bacterial transcriptome studies.

\section{Conclusions}

A simple method has been developed for making chromosomal uidA transcriptional reporter fusions in $S$. meliloti. The method is based on the narrow-host-range, high copy number, transcriptional uidA fusion selective pVMG for the effectiveness of the fusion construction. The fusions are verified by a simple colony PCR and a PCR product could be used to identify the fusion site by direct DNA sequencing. The method is successfully used in the model rhizobia S. meliloti. The method is also applicable to many other rhizobia stains, but it is not applicable to those that are resistant to both neomycin and ampicillin.

\section{Additional Files}

Additional File 1: The DNA sequence of the 720-bp PCR product. (DOCX 3534 kb)

Additional File 2: Southern blot of DNAs of S. meliloti strains. (DOCX 5227 kb) 


\section{Acknowledgments}

We thank Dr. Valerie Oke for advices and sending us pVO155. We thank Dr. Derek $\mathrm{H}$. Wells for sharing experience of designing PCR primers for the fusion verification. We thank Dr. Jianping Wang for helpful discussions. This work is supported by Agriculture and Food Research Initiative (AFRI) [award no. 2015-67013-22837/project accession no. 1005167] from the USDA National Institute of Food and Agriculture.

\section{Funding}

This work is supported by Agriculture and Food Research Initiative (AFRI) [award no. 2015-67013-22837/project accession no. 1005167] from the USDA National Institute of Food and Agriculture and by University of Florida's Institute of Food and Agricultural Science summer internships to A. Benge and R. Javier.

\section{Availability of Data and Materials}

$$
\text { Not applicable. }
$$

\section{Authors' Contributions}

MG designed this project and analyzed data. MG and TW wrote the manuscript. MG, $A B$, TW, and RJ conducted the experiments. All authors reviewed and approved the final manuscript.

\section{Ethics Approval and Consent to Participate}

$$
\text { Not applicable. }
$$

\section{Consent for Publication}

Not applicable.

\section{Competing Interests}

The authors declare that they have no competing interests.

\section{Publisher's Note}

Springer Nature remains neutral with regard to jurisdictional claims in published maps and institutional affiliations.

\section{Received: 7 February 2019 Accepted: 25 April 2019}

Published online: 03 May 2019

\section{References}

1. Oke V, Long SR. Bacterial genes induced within the nSodule during the Rhizobium-legume symbiosis. Mol Microbiol. 1999;32(4):837-49.

2. Wells DH, Long SR. The Sinorhizobium meliloti stringent response affects multiple aspects of symbiosis. Mol Microbiol. 2002;43(5):1115-27.

3. Gao M, Teplitski M. RIVET-a tool for in vivo analysis of symbiotically relevant gene expression in Sinorhizobium meliloti. Mol Plant-Microbe Interact. 2008:21(2):162-70.

4. Cowie A, Cheng J, Sibley CD, Fong Y, Zaheer R, Patten CL, Morton RM, Golding GB, Finan TM. An integrated approach to functional genomics: construction of a novel reporter gene fusion library for Sinorhizobium meliloti. Appl Environ Microbiol. 2006;72(11):7156-67.

5. Becker A, Schmidt M, Jager W, Puhler A. New gentamicin-resistance and lacZ promoter-probe cassettes suitable for insertion mutagenesis and generation of transcriptional fusions. Gene. 1995;162(1):37-9.

6. Jefferson RA, Burgess SM, Hirsh D. Beta-Glucuronidase from Escherichia coli as a gene-fusion marker. Proc Natl Acad Sci U S A. 1986;83(22):8447-51.

7. Parke D. Construction of mobilizable vectors derived from plasmids RP4, pUC18 and pUC19. Gene. 1990;93(1):135-7.

8. Kalogeraki VS, Winans SC. Suicide plasmids containing promoterless reporter genes can simultaneously disrupt and create fusions to target genes of diverse bacteria. Gene. 1997;188(1):69-75.

9. D'Haeze W, Holsters M. Nod factor structures, responses, and perception during initiation of nodule development. Glycobiology. 2002;12(6):79R-105R.

10. Pellock BJ, Teplitski M, Boinay RP, Bauer WD, Walker GC. A LuxR homolog controls production of symbiotically active extracellular polysaccharide II by Sinorhizobium meliloti. J Bacteriol. 2002;184(18):5067-76.

11. Galibert F, Finan TM, Long SR, Puhler A, Abola P, Ampe F, BarloyHubler F, Barnett MJ, Becker A, Boistard P, et al. The composite genome of the legume symbiont Sinorhizobium meliloti. Science. 2001;293(5530):668-72.

12. Gao M, Chen H, Eberhard A, Gronquist MR, Robinson JB, Rolfe BG, Bauer WD. sinl- and expR-dependent quorum sensing in Sinorhizobium meliloti. J Bacteriol. 2005;187(23):7931-44.

13. Friedman AM, Long SR, Brown SE, Buikema WJ, Ausubel FM. Construction of a broad host range cosmid cloning vector and its use in the genetic analysis of Rhizobium mutants. Gene. 1982;18(3):289-96.

14. Beringer JE. R factor transfer in Rhizobium leguminosarum. J Gen Microbiol. 1974:84(1):188-98.

15. Bittner AN, Oke V. Multiple groESL operons are not key targets of RpoH1 and RpoH2 in Sinorhizobium meliloti. J Bacteriol. 2006;188(10):3507-15.

16. Maniatis T, Fritsch EF, Sambrook J. Molecular cloning: a laboratory manual. Cold Spring Harbor: Cold Spring Harbor Laboratory; 1982.

17. Gao MBA, Mesa JM, Javier R, Liu FX. Use of RNA Immunoprecipitation method for determining Sinorhizobium meliloti RNA-Hfa protein associations in vivo. Biol Proced Online. 2018:20:8.

18. Gao M, Chen H, Eberhard A, Gronquist MR, Robinson JB, Connolly M, Teplitski M, Rolfe BG, Bauer WD. Effects of AiiA-mediated quorum quenching in Sinorhizobium meliloti on quorum-sensing signals, proteome patterns, and symbiotic interactions. Mol Plant-Microbe Interact. 2007;20(7):843-56.

19. Gao M, Nguyen H, Salas Gonzalez I, Teplitski M. Regulation of fix LJ by Hfa controls symbiotically important genes in Sinorhizobium meliloti. Mol PlantMicrobe Interact. 2016;29(11):844-53.

20. Marketon MM, Gronquist MR, Eberhard A, Gonzalez JE. Characterization of the Sinorhizobium meliloti $\operatorname{sinR/\operatorname {sin}l~locus~and~the~production~of~novel~} \mathrm{N}$-acyl homoserine lactones. J Bacteriol. 2002;184(20):5686-95.

21. Mclntosh M, Krol E, Becker A. Competitive and cooperative effects in quorum-sensing-regulated galactoglucan biosynthesis in Sinorhizobium meliloti. J Bacteriol. 2008;190(15):5308-17.

22. D'Haeze W, Verplancke C, Mironov V, Holsters M. pMH11, a tool for gene disruption and expression analysis in Azorhizobium caulinodans. Plasmid. 2002;47(2):88-93.

23. Southern EM. Detection of specific sequences among DNA fragments separated by gel electrophoresis. J Mol Biol. 1975;98(3):503-17.

24. Gao M, Barnett MJ, Long SR, Teplitski M. Role of the Sinorhizobium meliloti global regulator $\mathrm{Hfa}$ in gene regulation and symbiosis. Mol Plant-Microbe Interact. 2010;23(4):355-65.

25. Llamas I, Keshavan N, Gonzalez JE. Use of Sinorhizobium meliloti as an indicator for specific detection of long-chain $\mathrm{N}$-acyl homoserine lactones. Appl Environ Microbiol. 2004;70(6):3715-23.

26. Griffitts JS, Carlyon RE, Erickson JH, Moulton JL, Barnett MJ, Toman CJ, Long SR. A Sinorhizobium meliloti osmosensory two-component system required for cyclic glucan export and symbiosis. Mol Microbiol. 2008;69(2):479-90.

27. Gurich N, Gonzalez JE. Role of quorum sensing in Sinorhizobium melilotialfalfa symbiosis. J Bacteriol. 2009;191(13):4372-82.

28. Silhavy TJ, Beckwith JR. Uses of lac fusions for the study of biological problems. Microbiol Rev. 1985;49(4):398-418.

29. Chalfie M, Tu Y, Euskirchen G, Ward WW, Prasher DC. Green fluorescent protein as a marker for gene expression. Science. 1994; 263(5148):802-5.

30. Tsien RY. The green fluorescent protein. Annu Rev Biochem. 1998;67:509-44.

31. Karunakaran R, Mauchline TH, Hosie AH, Poole PS. A family of promoter probe vectors incorporating autofluorescent and chromogenic reporter proteins for studying gene expression in gram-negative bacteria. Microbiology. 2005;151(Pt 10):3249-56.

32. Price PA, Tanner HR, Dillon BA, Shabab M, Walker GC, Griffitts JS. Rhizobial peptidase HrrP cleaves host-encoded signaling peptides and mediates symbiotic compatibility. Proc Natl Acad Sci U S A. 2015; 112(49):15244-9.

33. Leenhouts KJ, Kok J, Venema G. Campbell-like integration of heterologous plasmid DNA into the chromosome of Lactococcus lactis subsp. lactis. Appl Environ Microbiol. 1989;55(2):394-400.

34. Gao M, D'Haeze W, De Rycke R, Wolucka B, Holsters M. Knockout of an azorhizobial dTDP-L-rhamnose synthase affects lipopolysaccharide and extracellular polysaccharide production and disables symbiosis with Sesbania rostrata. Mol Plant-Microbe Interact. 2001;14(7):857-66.

35. Oke V, Long SR. Bacteroid formation in the Rhizobium-legume symbiosis. Curr Opin Microbiol. 1999;2(6):641-6. 
36. Camilli A, Beattie DT, Mekalanos JJ. Use of genetic recombination as a reporter of gene expression. Proc Natl Acad Sci U S A. 1994;91(7):2634-8.

37. Hsu PD, Lander ES, Zhang F. Development and applications of CRISPR-Cas9 for genome engineering. Cell. 2014;157(6):1262-78.

38. Jiang W, Bikard D, Cox D, Zhang F, Marraffini LA. RNA-guided editing of bacterial genomes using CRISPR-Cas systems. Nat Biotechnol. 2013;31(3):233-9.

Ready to submit your research? Choose BMC and benefit from:

- fast, convenient online submission

- thorough peer review by experienced researchers in your field

- rapid publication on acceptance

- support for research data, including large and complex data types

- gold Open Access which fosters wider collaboration and increased citations

- maximum visibility for your research: over $100 \mathrm{M}$ website views per year

At $\mathrm{BMC}$, research is always in progress.

Learn more biomedcentral.com/submissions 